Computer Optics and Nanophotonics

\title{
FORMATION OF PLASMONIC NANOJETS BY SILVER NANO-STRIP
}

\author{
E.S. Kozlova \\ Image Processing Systems Institute - Branch of the Federal Scientific Research Centre "Crys- \\ tallography and Photonics" of Russian Academy of Sciences, Samara, Russia, \\ Samara National Research University, Samara, Russia
}

\begin{abstract}
In this work the "central" surface plasmon-polariton was obtained by using frequency dependent difference time-domain method for the TMpolarized light at $532 \mathrm{~nm}$, which was propagating through the silver nano-strip, placed on silica glass in an aqueous medium. The height and width of nanostrip was equal to $20 \mathrm{~nm}$ and $215 \mathrm{~nm}$ respectively. The intensity of surface plasmon-polariton was 4 times higher the intensity of the incident radiation. The full width at half maximum of the nanojet was $138 \mathrm{~nm}$.
\end{abstract}

Keywords: surface plasmon-polariton, nano-strip, (FD) ${ }^{2}$ TD-method.

Citation: Kozlova ES. Formation of plasmonic nanojets by silver nano-strip. CEUR Workshop Proceedings, 2016; 1638: 1-7. DOI: 10.18287/1613-00732016-1638-1-7

\section{Introduction}

Today great attention is paid to such optical phenomena as surface plasmon-polaritons (SPPs) which arise during interaction of light with the metal and propagate along the interfaces between metal and dielectric [1-2]. SPP can be widely used to solve various problems of modern science and technology [3-7]. A large number of works devoted to the modeling of nano-antennas, based on SPP's excitation effect [8-9]. A FabryPerot model was formulated that predicts both the peak position and spectral shape of optical resonances for short-range SPP. The authors used full-field simulation based on the finite-difference time-domain method (FDTD-method) to calculate the parameters for this model [8]. Whereas, some other authors used rectangular gold and silver nano-strips embedded in glass or water. The effect of SPP's resonance is analyzed using a surface integral equation method. They showed the feasibility of at least 10fold field magnitude enhancement for local field in narrow $(5 \mathrm{~nm})$ gap between two metal strips [9]. But all these authors didn't investigate another properties of SPP like length and full width at half maximum (FWHM).

In our previous work we've investigated the amplitude Fresnel zone plate for focusing of laser light. During this study we've found SPP's on surface of silver relief of this zone plate [9]. In this paper, we used the frequency depended FDTD-method 
((FD) ${ }^{2}$ TD-method) for investigating the process of SPP's formation on silver nanostrip for the incident TM-polarized light at $532 \mathrm{~nm}$. We estimated spatial characteristics such as length and FWHM of SPPs which is new investigation up to our knowledge. In contrast to the works described above, silver strips are placed on a silica glass, which has a high influence on the process of SPP's formation.

\section{Formation of SPPs on silver nano-strip}

We considered the propagation of a TM-polarized light at $532 \mathrm{~nm}$, which was normally incident on the metal nano-strip placed on a substrate. The optical scheme is presented on Figure 1. Height and width of nano-strip are $h$ and $w$ respectively. The permittivity of the medium, nano-strip and substrate are $\varepsilon_{1}, \varepsilon_{m}$ and $\varepsilon_{2}$ respectively. The light is propagating along $Z$ axis. All the simulations were carried out by FullWAVE (RSoft) based on (FD) ${ }^{2}$ TD-method. Hereinafter, the following simulation parameters were used: steps in space were $2 \mathrm{~nm}$, time step was $1 \mathrm{~nm}(c T$, where $c$ is speed of light in vacuum, $T$ is period of light).

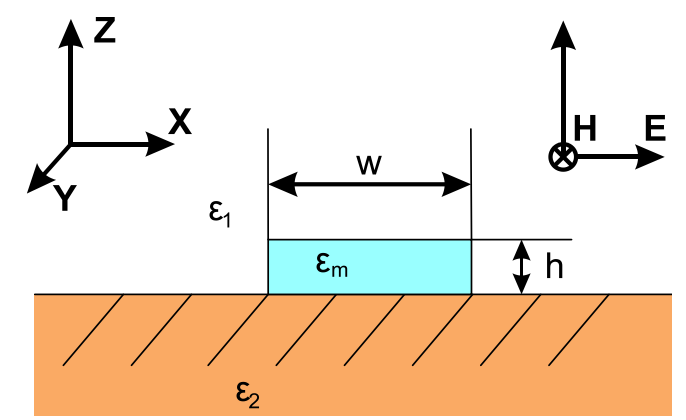

Fig. 1. Optical scheme for nano-strip

Silica glass was considered as a material of substrate. Table 1 shows parameters for the Sellmeier's permittivity model of silica glass [11]:

$\varepsilon_{2}(\lambda)=\varepsilon_{\infty}+\sum_{m} \frac{\Delta \varepsilon_{m} \lambda^{2}}{\lambda^{2}-\lambda_{m}^{2}-i \lambda \eta_{m}}$,

Where $\lambda$ is a wavelength; $\varepsilon_{\infty}(x, z)$ is the permittivity in the limit of infinite frequency; $\Delta \varepsilon_{m}$ is the resonator strength; $\lambda_{m}$ is the resonant wavelength; $\eta_{m}$ is the Sellmeier damping factor.

Table 1. Parameters for the Sellmeyer's permittivity model of silica glass

\begin{tabular}{cccc}
\hline $\boldsymbol{m}$ & $\boldsymbol{\Delta} \boldsymbol{\varepsilon}_{\boldsymbol{m}}, \boldsymbol{\mu m}$ & $\lambda_{\boldsymbol{m}}, \boldsymbol{\mu m}$ & $\boldsymbol{\delta}_{\boldsymbol{m}}, \boldsymbol{\mu m}$ \\
\hline $\mathbf{1}$ & 0.69616630 & 0.068404300 & 0 \\
$\mathbf{2}$ & 0.40794260 & 0.11624140 & 0 \\
$\mathbf{3}$ & 0.89747940 & 9.8961610 & 0 \\
& & $\boldsymbol{\varepsilon}_{\infty}=\mathbf{1}$ & \\
\hline
\end{tabular}


Silver was considered as a material of nano-strip. Table 2 shows parameters for the Drude-Lorentz's permittivity model of silver [12]:

$$
\varepsilon_{m}(\omega)=\varepsilon_{\infty}+\frac{\omega_{p}^{2}}{-2 i \omega v-\omega^{2}}+\sum_{m} \frac{A_{m} \omega_{m}^{2}}{-\omega^{2}-2 i \omega \delta_{m}+\omega_{m}^{2}}
$$

Where $\omega$ is a frequency; $\omega_{p}$ is the plasma frequency; $v$ is the collision frequency; $A_{m}$ is the resonator strength; $\delta_{m}$ is the damping factor; $\omega_{m}$ is the resonant frequency.

Table 2. Parameters for the Drude-Lorentz's permittivity model of silver

\begin{tabular}{cccc}
\hline $\boldsymbol{m}$ & $\mathbf{A}_{\boldsymbol{m}}, \mathbf{H z}$ & $\boldsymbol{\delta}_{\boldsymbol{m}}, \mathbf{H z}$ & $\boldsymbol{\omega}_{\boldsymbol{m}}, \mathbf{H z}$ \\
\hline $\mathbf{1}$ & 7.924697 & 19.68071 & 4.132646 \\
$\mathbf{2}$ & 0.501327 & 2.289161 & 22.6941 \\
$\mathbf{3}$ & 0.013329 & 0.329194 & 41.45307 \\
$\mathbf{4}$ & 0.826552 & 4.639097 & 46.001 \\
$\mathbf{5}$ & 1.113336 & 12.25 & 102.759 \\
& & &
\end{tabular}

$$
\begin{gathered}
\omega_{p}=41.94605 \mathrm{~Hz} \\
v=0.243097 \mathrm{~Hz}
\end{gathered}
$$

For the first series of simulation we fixed the strip height equals to $h=20 \mathrm{~nm}$, remove the substrate and choose air $\left(\varepsilon_{l}=1\right)$ as a medium. After each calculation, we measured the maximum intensity of light at $2 \mathrm{~nm}$ above the nano-strip. Figure 2 shows the SPP's intensity dependence on nano-strip width.

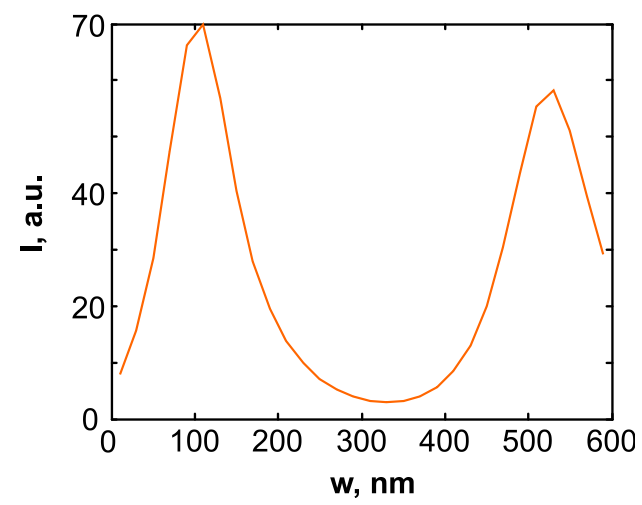

Fig. 2. The SPP's intensity dependence on nano-stip width

By comparing the simulation results for light propagation through the silver nanostrip of various widths, the resonant width $w=110 \mathrm{~nm}$ has been selected. Nano-strip with this width forms a "boundary" SPPs with the highest value of the intensity in the central peak. Figure 3 shows the intensity distribution obtained at $2 \mathrm{~nm}$ above silver nano-strip with width of $w=110 \mathrm{~nm}$. 


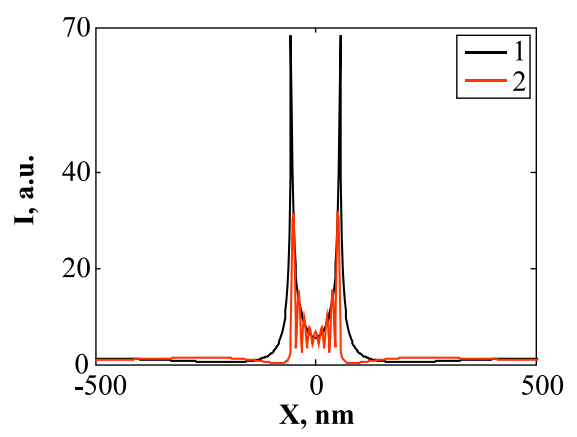

Fig. 3. Intensity distribution at $2 \mathrm{~nm}$ above silver nano-strip of width , $w=110 \mathrm{~nm}$ placed in air (line 1) and on the silica glass placed in water (line 2)

Figure 3 shows two powerful "boundary" SPP (line 1) with high intensity (68 a.u.) and small FWHM $(8 \mathrm{~nm})$. Figure 4 shows the intensity distribution in the SPP along axis of its propagation. Measurement of SPPs "length" showed that the distance of decline to incident intensity through the $\mathrm{Z}$ direction is $68 \mathrm{~nm}$, while through the direction of SPPs propagation it is $94 \mathrm{~nm}$ (Figure 4, line 1). The inclination angles of straight which were coinciding with the directions of SPPs propagation are $112^{\circ}$ and $68^{\circ}$.

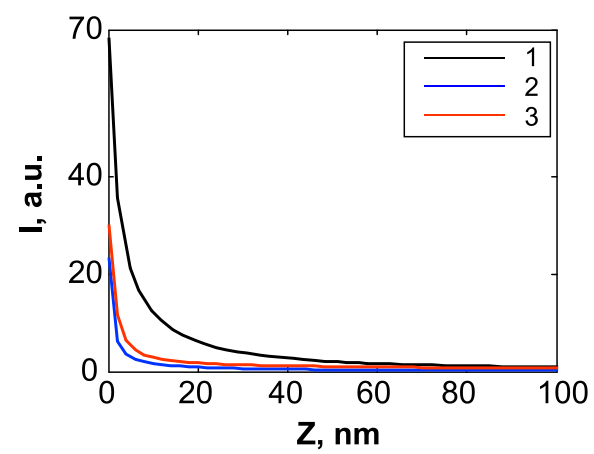

Fig. 4. SPP's intensity distribution through the direction of SPP's propagation in the case of using nano-strip placed in air (line 1) and nano-strip on the silica glass placed in air (line 2) and water (line 3)

For the next simulations the substrate from silica glass was added. In this case, the direction of SPPs propagation changed and practically coincided with the direction of light propagation (it was parallel to the $\mathrm{Z}$ axis). However, the presence of the substrate substantially disrupts the formation of the SPP. It leads to fluctuations at the interface substrate/strip environments, reduces the maximum intensity and "length" till $20 \mathrm{~nm}$ (Figure 4, line 2) of "boundary" SPPs.

To compensate this effect, water $\left(\varepsilon_{l}=1.78\right)$ was chosen as a main medium instead of air. In this case measurement of SPPs "length" showed that the distance of decline to incident intensity through the $\mathrm{Z}$ direction is $56 \mathrm{~nm}$ (Figure 4, line 3). FWHM of 
"boundary" SPPs is $75 \mathrm{~nm}$ (Figure 3, line 2). The intensity of "boundary" SPPs is 36 times higher than intensity of incident light (Figure 3, line 2). However, the use of such sharply focused light is complicated by the close proximity of these peaks to each other and the presence of the boundary plasmonic lobes, which are formed along the entire nano-strip.

In addition to "boundary" short-range SPPs which have a maximum intensity, there are other SPPs. However, during the study of dependences of SPP's intensity distribution on the width of nano-strip placed in the air shows no possibility to obtain a "long" single SPP in the center of nano-strip. A similar study for the nano-strip placed on the silica glass in an aqueous medium revealed the presence of this "central" SPP. By comparing the simulation results for light propagation through the silver nano-strip of various widths, the resonant width $\mathrm{w}=215 \mathrm{~nm}$ has been selected. Figure 5 shows the intensity distribution at $4 \mathrm{~nm}$ above silver nano-strip with width of $\mathrm{w}=215 \mathrm{~nm}$.

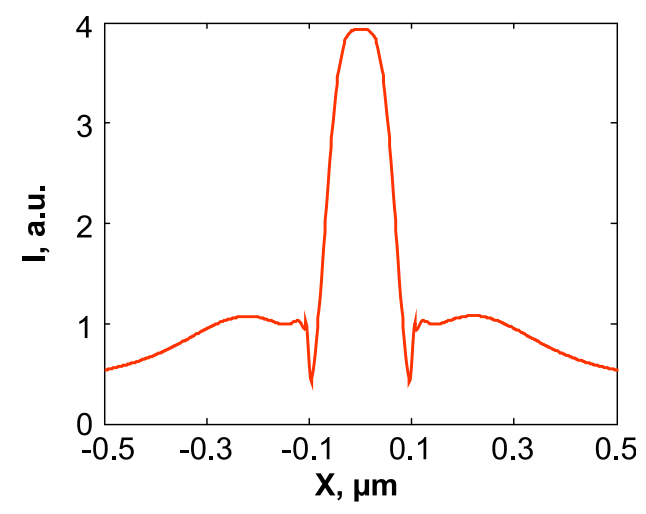

Fig. 5. Intensity distribution $4 \mathrm{~nm}$ after silver nano-strip with width of $\mathrm{w}=215 \mathrm{~nm}$ on the silica glass placed in water

Figure 5 shows that the "boundary" SPPs is almost completely absent. FWHM of "central" SPP is $138 \mathrm{~nm}$. The intensity of "central" SPPs is four times higher than intensity of incident light (Figure 5). The measurement of SPPs "length" showed that the decay length to incident intensity through $\mathrm{Z}$ direction is $54 \mathrm{~nm}$. The "central" SPP is also observed on the silver nano-strip with width of $\mathrm{w}=215 \mathrm{~nm}$ placed on silica glass in water, but "boundary" SPPs are also present and their intensity is comparable with the intensity of "central" SPP.

\section{Conclusion}

In this work the process of SPPs formation on silver nano-strip for TM-polarized light at $532 \mathrm{~nm}$ is studied. The spatial characteristics of SPPs like "length" and FWHM were investigated by using (FD) ${ }^{2}$ TD-method. In contrast to the $[8,9]$, silver nanostrips are placed on a silica glass, the presence of which brings a disturbance in the process of SPP's formation. It leads to fluctuations at the interface substrate/strip envi- 
ronments, reduces the maximum intensity and "length" till $20 \mathrm{~nm}$ (Figure 4, line 2) of "boundary" SPPs. To compensate this effect, the water $\left(\varepsilon_{l}=1.78\right)$ was chosen as a main medium instead of air. In this case measurement of SPPs "length" showed that the decay length to incident intensity through the $\mathrm{Z}$ direction is $56 \mathrm{~nm}$ (Figure 4, line 3). FWHM of "boundary" SPPs is $75 \mathrm{~nm}$ (Figure 3, line 2). The intensity of "boundary" SPPs is 36 times higher than intensity of incident light (Figure 3, line 2). Simulations by (FD) ${ }^{2}$ TD-method showed presence of "central" SPP for silver nano-strip placed on silica glass in water. FWHM of "central" SPP is $138 \mathrm{~nm}$. The intensity of "central" SPPs is four times higher than the intensity of incident light (Figure 5). The "boundary" SPPs is almost completely absent. The results can be used to design devices that allow capturing and moving the particles in water or other biofluidics [1314].

\section{Acknowledgements}

We acknowledge funding from the Russian Foundation for Basic Research (RFBR) (16-07-00990,14-29-07133, 15-47-02492, 15-37-20723, 15-07-01174, 14-07-97039), the Ministry of Education and Science of the Russian Federation (and also MK-9019.2016.2, SP-4375.2016.5).

\section{References}

1. Bezus EA, Doskolovich LL. Phase modulation and refraction of surface plasmon polaritons with parasitic scattering suppression. Computer Optics, 2014; 38(4): 623-628. [In Russian].

2. Soifer VA, Kotlyar VV, Doskolovich LL. Diffractive optical elements in nanophotonics devices. Computer Optics, 2009; 33(4): 352-368. [In Russian]

3. Kadomina EA, Bezus EA, Doskolovich LL. Resonant photonic-crystal structures with a diffraction grating for refractive index sensing. Computer Optics, 2016; 40(2): 164-172. [In Russian]. DOI: 10.18287/2412-6179-2016-40-2-164-172.

4. Ma R-M, Oulton RF, Sorger VJ and Zhang X. Plasmon lasers: coherent light source at molecular scales. Laser \& Photonics Reviews, 2013; 7:1-21.

5. Xie Z, Yu W, Wang T, Zhang H, Fu Y, Liu H, Li F, Lu Z and Sun Q. Plasmonic Nanolithography: A Review. Plasmonics, 2011; 6: 565-580.

6. Han Z and Bozhevolnyi SI. Radiation guiding with surface plasmon polaritons. Reports on Progress in Physics, 2013; 76: 016402.

7. Atwater HA and Polman A. Plasmonics for improved photovoltaic devices. Nature Materials, 2010; 9: 205-213.

8. Barnard ES, White JS, Chandran A, and Brongersma ML. Spectral properties of plasmonic resonator antennas. Optics Express, 2008; 16(21): 16529-16537.

9. Søndergaard T. and Bozhevolnyi S.I. Metal nano-strip optical resonators. Optics Express, 2008; 15(7): 4198-4204.

10. Kozlova ES, Kotlyar VV, Nalimov AG. Comparative modeling of amplitude and phase zone plates. Computer Optics, 2015; 39(5): 687-693. [In Russian]. DOI: 10.18287/01342452-2015-39-5-687-693. 
11. Couairon A. Sudrie L, Franco M, Prade B, Mysyrowicz A. Filamentation and damage in fused silica induced by tightly focused femtosecond laser pulses. Physical Review B: Condensed Matter, 2005; 71(12): 125435-125441.

12. Vial A, Laroche T, Dridi M, and Le Cunff L. A new model of dispersion for metals leading to a more accurate modeling of plasmonic structures using the FDTD method. Applied Physics A, 2011; 103(3): 849-853.

13. Porfirev AP, Kovalev AA, Kotlyar VV. Optical trapping and moving of microparticles using asymmetrical bessel-gaussian beams. Computer Optics, 2016; 40(2): 152-157. [In Russian]. DOI: 10.18287/2412-6179-2016-40-2-152-157.

14. Porfirev AP, Skidanov RV. Optical capture of microparticles in special traps. Computer Optics, 2012; 36(2): 211-218. [In Russian] 